\title{
Tracking Impact of Interstitial Lung Disease in Systemic Sclerosis in a Complete Nationwide Cohort
}

\author{
Anna-Maria Hoffmann-Vold ${ }^{1,2}$, Havard ${ }^{\circ}$ Fretheim $^{1,2}$, Anne-Kristine Halse ${ }^{3}$, Marit Seip ${ }^{4}$, Helle Bitter ${ }^{5}$, \\ Marianne Wallenius ${ }^{6,7}$, Torhild Garen ${ }^{1}$, Anne Salberg ${ }^{8}$, Cathrine Brunborg ${ }^{9}$, Øyvind Midtvedt ${ }^{1}$, May Brit \\ Lund $^{2,10}$, Trond M. Aaløkken ${ }^{11}$, and Øyvind Molberg ${ }^{1,2}$ \\ ${ }^{1}$ Department of Rheumatology, ${ }^{9}$ Oslo Centre for Biostatistics and Epidemiology, Research Support Services; ${ }^{10}$ Department of \\ Respiratory Medicine, and ${ }^{11}$ Department of Radiology and Nuclear Medicine, Oslo University Hospital, Oslo, Norway; ${ }^{2}$ Institute of \\ Clinical Medicine, University of Oslo, Oslo, Norway; ${ }^{3}$ Department of Rheumatology, Haukeland University Hospital, Bergen, \\ Norway; ${ }^{4}$ Department of Rheumatology, University Hospital of North Norway, Tromso, Norway; ${ }^{5}$ Department of Rheumatology, Hospital \\ of Southern Norway, Kristiansand, Norway; ${ }^{6}$ Norwegian National Advisory Unit of Pregnancy and Rheumatic Diseases, Department of \\ Rheumatology, Trondheim University Hospital, Trondheim, Norway; ${ }^{7}$ Institute of Neuromedicine and Movement Science, Norwegian \\ University of Science and Technology, Norwegian University of Science and Technology, Trondheim, Norway; and ${ }^{8}$ Department of \\ Rheumatology, Lillehammer Hospital, Lillehammer, Norway
}

\section{Abstract}

Rationale: Interstitial lung disease (ILD) represents a major challenge in systemic sclerosis (SSc), but there are no precise, population-based data on its overall impact, limiting opportunities for screening and management strategies.

Objectives: Evaluate impact of ILD in a unique, nationwide, population-based SSc cohort.

Methods: ILD was assessed prospectively in the Norwegian SSc (Nor-SSc) cohort, including all 815 patients with SSc resident in the country from 2000 to 2012. Lung high-resolution computed tomography (HRCT) scans were available for fibrosis quantification at baseline ( $n=650,80 \%)$ and follow-up. Pulmonary function tests were assessed at baseline $(n=703,86 \%)$ and follow-up. Vital status and standardized mortality ratios (SMRs) were estimated at study end (2018) in the 630 incident Nor-SSc cases and 15 individually matched control subjects. Cumulative survival rates were computed.
Measurements and Main Results: At baseline, $50 \%$ of the subjects with SSc $(n=324)$ had ILD by HRCT and $46 \%$ displayed pulmonary function declines consistent with ILD progression. Mortality correlated with extent of lung fibrosis as SMR increased from 2.2 with no fibrosis to 8.0 with greater than $25 \%$ fibrosis. SMR was inversely related to baseline $\mathrm{FVC} \%$ and increased at all FVC levels below $100 \%$. In patients with normal-range baseline FVC (80-100\%), the 5and 10-year survival rates correlated with presence or absence of lung fibrosis, being $83 \%$ and $80 \%$, respectively, with no fibrosis and $69 \%$ and $56 \%$, respectively, with lung fibrosis $(P=0.03)$.

Conclusions: The mere presence of ILD at baseline appears to affect outcome in SSc, suggesting that all patients with SSc should undergo a baseline pulmonary function test and lung HRCT screening to diagnose ILD early and tailor further management.

Keywords: systemic sclerosis; epidemiology; pulmonary fibrosis; autoimmune disease
Systemic sclerosis (SSc) is a devastating, heterogeneous, multiorgan inflammatory disease characterized by uncontrolled fibrosis of skin and internal organs, vascular pathology leading to small vessel obliteration, and distinct serum autoantibodies (1-3). SSc associates with high disease burden and reduced life expectancy, with interstitial lung disease (ILD) and pulmonary hypertension (PH) as major causes of disease-related deaths (48). Although ILD undoubtedly is a

Supported by the Norwegian Women's Public Health Association and the South Eastern Regional Health Authorities.

Author Contributions: Conception and design: A.-M.H.-V. and Ø. Molberg. Analysis and interpretation: A.-M.H.-V., H.F., A.-K.H., H.B., M.S., M.W., A.S., C.B., T.G., Ø. Midtvedt, M.B.L., T.M.A., and Ø. Molberg. Drafting the manuscript for important intellectual content and final approval of the version to be published: all authors.

Correspondence and requests for reprints should be addressed to Anna-Maria Hoffmann-Vold, M.D., Ph.D., Oslo University Hospital, Rikshospitalet, Pb 4950 Nydalen, 0424 Oslo, Norway. E-mail: a.m.hoffmann-vold@medisin.uio.no. 


\section{At a Glance Commentary}

Scientific Knowledge on the Subject: Interstitial lung disease (ILD) represents a major challenge in systemic sclerosis (SSc); despite the need for screening and management recommendations for ILD in patients with $\mathrm{SSc}$, these are lacking. To date, there are no precise, population-based data on its overall impact that may help developing these.

What This Study Adds to the Field:

We show in a unique nationwide SSc cohort that the mere presence of ILD at SSc diagnosis appears to affect outcome in SSc. Even SSc-ILD patients with mild lung fibrosis and normal range FVC developed frequently progressive ILD and had reduced survival. The combination of normal range FVC and no fibrosis on high-resolution computed tomography at baseline conferred a better prognosis. It appears rational that all patients with SSc should undergo screening with baseline pulmonary function tests and lung high-resolution computed tomography, including detailed reports of the extent of fibrosis at ILD diagnosis in order to diagnose ILD early, assess risk, and initiate treatment when indicated and tailor further management. Importantly, SScILD patients with mild lung fibrosis and normal range FVC developed frequently progressive ILD, and even moderate ILD progression after long disease duration was associated with worse outcome. Thus, it appears critical to monitor all patients with SSc using regular pulmonary function tests and to pay close attention to trending declines, particularly in cases with known SScILD at baseline.

challenge in SSc, there are no precise, population-based data on its overall impact, limiting future development of screening and management strategies (3, 9-11).

High-resolution computed tomography (HRCT) is the primary tool to diagnose ILD and determine patterns of ILD-related abnormalities and the extent of lung fibrosis (12-16). Despite SSc being a significant risk factor for ILD, there are no existing guidelines on whether patients with SSc should undergo a lung HRCT at time of $\mathrm{SSc}$ diagnosis. Nor are there any definitions or threshold values for the extent of HRCT changes needed to diagnose SSc-ILD.

Higher extent of lung fibrosis measured by visual scoring has been shown to associate with short-term decline in lung function and with increased mortality, whereas limited extent of lung fibrosis is associated with progression; however, its relation to survival is unknown (7, 17-19). Lastly, it appears that an early HRCT without lung fibrosis is associated with very low risk for later development of SSc-ILD (16).

No formalized guidelines for ILD screening in SSc exist, but there is agreement among SSc experts that benchmarking of lung function with pulmonary function tests (PFTs) at time of SSc diagnosis is important and critical to estimate the severity of ILD and to predict outcome in SSc-ILD $(7,16$, 19). However, prospective cohort data indicate poor performance of PFT as a standalone method for ILD screening in SSc (20, $21)$. Not surprisingly, a low FVC $(, 70 \%)$ at baseline has been shown to be associated with increased mortality, but there are no data on potential relationship between intermediary $\mathrm{FVC} \%$ values and mortality (22-24). This is probably related to the wide normal ranges of FVC and other PFT parameters, and frequently missing premorbid data, making it difficult to interpret baseline PFT values that are within, or slightly below, the normal range $(16,20$, 21, 25). Repeated PFTs are essential for follow-up and management strategies for patients with SSc and strategies are mainly adopted from idiopathic pulmonary fibrosis (26-29).

There are several key research questions that can only be approached by studying unselected cohorts from welldefined study areas, including equality of care, implementation of evidence-based guidelines or recommendations, and the impact these guidelines actually have on critical outcome measures. The Scandinavian countries, including Norway, are among the few countries in the world where it is possible to create complete population-based cohorts on rare disease like $\mathrm{SSc}$, as previously described in regional studies $(30,31)$. Briefly, this opportunity is given by three societal factors: 1) all patients with SSc are followed at public hospitals and therefore easily identified, 2) the mandatory national ID numbers secure against loss to follow-up, and 3) the possibility of linking patient cohorts to national registries makes it possible to check vital status at any time and to identify causes of death $(31,32)$.

In the present study, we created a key method for unbiased work on prognostic and predictive factors of ILD involvement in SSc by including all patients with SSc in Norway in a prospective, nationwide Norwegian SSc (Nor-SSc) cohort to determine impact on mortality.

\section{Methods}

\section{The Nationwide Nor-SSc Cohort}

All patients with SSc resident in Norway between 2000 and 2012 were captured by a stepwise strategy. In the first step, performed in 2013, we searched through the administrative databases of all public hospitals in Norway, and of all private rheumatologists in the country, to identify all patient contacts coded by the International Statistical Classification of Diseases and Related Health Problems, 10th revision (used in Norway since 1999), as M34.0, M34.1, M34.8 and M34.9 (SSc) at least once during the study period. In the second step, performed from 2013 to 2016, we reviewed in detail the electronic patient charts (available for all patients) of each identified case. Chart notes from time of diagnosis before establishment of electronic journals have mostly been scanned and were also available in the electronic charts. Each M34 code identified in the databases was checked thoroughly and validated by the first author, an SSc expert. Relevant parameters in these patients were recorded, including clinical features, demographic data, autoantibody status, and treatment with immunemodulating agents. All included patients with $\mathrm{SSc}$ in the Nor-SSc cohort were older than 18 years, had a clinical SSc diagnosis made by an expert, and met the 2013 American College of Rheumatology-European League Against Rheumatism SSc classification criteria (33). Briefly, these criteria are based on a scoring system in which the first criterion alone, skin thickening of the fingers of both hands extending proximal to the metacarpophalangeal joints, gives the 9 points needed to classify the patient as SSc. If the first criterion is not met, the patient can gain 9 points by seven other items (33). Incident cases were diagnosed from January 
1, 2000, to December 31, 2012, whereas prevalent cases included patients diagnosed before 2000 .

This study complied with the Declaration of Helsinki. The Regional Committee of Health and Medical Research Ethics South East in Norway approved the study and received exemption of informed consent for identification of the patients and chart review (No. 2009/1035). Approval was given to link patients' clinical data to the registry of causes of deaths and the national population registry to receive random control subjects.

\section{Assessment of ILD and $\mathrm{PH}$}

PFTs and electronic lung HRCT image files from baseline and last available follow-up visit were retrieved (16). All HRCT scans were reviewed manually, independently, and in random order by one experienced chest radiologist and one rheumatologist with training in interpretation of HRCT scans, both blinded to lung function and the patient's clinical condition, as previously described (16). Briefly, we defined reticular pattern abnormalities and superimposed ground-glass opacities as equivalent to fibrosis (34), evaluated extent of fibrosis independently in 10 thin-section images, and assigned a score for each image based on the percentage of parenchyma with fibrotic changes. Area measurements were done precisely by drawing a freehand region of interest on the picture archiving and communication system (PACS) screen. The relationship between the overall volume of fibrosis and total lung volume was evaluated for HRCT at baseline and at follow-up. Fibrosis extent was expressed as percentage of total lung volume (16). To assess the sequential changes over time for each patient, the percentage rate of abnormal HRCT findings were compared with percentage rates on the other HRCT scan. Progression of fibrosis was expressed as increasing extent of fibrosis from baseline to follow-up HRCT.
PFTs with DLCO, FVC, and FEV 1 were performed according to American Thoracic Society-European Respiratory Society guidelines as described $(16,35)$. The course of ILD during the observation period was assessed by absolute changes in percentage predicted from baseline to follow-up, and defined as severe ILD progression (total FVC decline $.10 \%$ or $5-10 \%$ with DLCO decline $>15 \%$ ), moderate ILD progression (FVC decline, 5-10\%), or stable FVC (65\% FVC change) $(19,36,37)$.

The 6-minute-walk distance test and symptoms of dyspnea measured by functional classes were also noted (38). PH was diagnosed by right heart catheterization (RHC), according to the updated European Society of Cardiology guidelines, as a mean pulmonary arterial pressure greater than or equal to $25 \mathrm{~mm}$ $\mathrm{Hg}$, measured with RHC (39). PH-ILD (World Health Organization [WHO] group 3) was defined as precapillary $\mathrm{PH}$ combined with findings of lung fibrosis

Table 1. Demographics, Treatment, Key Systemic Sclerosis Features, and Organ Involvement in the Total Norwegian Systemic Sclerosis Cohort and Segregated by Extent of Interstitial Lung Disease

\begin{tabular}{|c|c|c|c|c|}
\hline & & & HRCT Available $(n=650)$ & \\
\hline Characteristics & Total $(n=815)$ & No ILD $(n=324)$ & $<10 \%$ ILD $(n=249)$ & $>10 \%$ ILD $(n=77)$ \\
\hline \multicolumn{5}{|l|}{ Demographics } \\
\hline Age at diagnosis, yr, mean (SD) & $53(14.8)$ & $54.5(15.0)$ & $52.5(14.5)$ & $53.1(15.1)$ \\
\hline Time onset to diagnosis, yr, mean (SD) & $3.0(5.1)$ & $3.3(5.4)$ & $3.3(5.6)$ & $2.4(3.2)$ \\
\hline Observation period, yr, mean (SD) & $11.1(8)$ & $14.0(9)$ & $11.0(6.8)$ & $10.5(8.1)$ \\
\hline Female sex & $682(84)$ & $283(87)$ & $181(73)$ & $53(69)$ \\
\hline Ever smoker & $440(54)$ & $162(50)$ & $137(55)$ & $37(48)$ \\
\hline Deceased & $192(24)$ & $61(19)$ & $93(37)$ & $38(49)$ \\
\hline \multicolumn{5}{|l|}{ Key ILD features } \\
\hline FVC, \% predicted, mean (SD) & $94(21)$ & $99(19)$ & $91(21)$ & $78(19)$ \\
\hline DLco, \% predicted, mean (SD) & $69(20)$ & $75(19)$ & $65(19)$ & $51(18)$ \\
\hline Functional class 3 and 4 & $74(9)$ & $23(7)$ & $26(10)$ & $25(33)$ \\
\hline 6-minute-walk distance, $\mathrm{m}$, mean (SD) & $457(150)$ & $462(150)$ & $459(162)$ & $435(121)$ \\
\hline \multicolumn{5}{|l|}{ Treatment } \\
\hline Cyclophosphamide & $67(8.2)$ & $11(3.4)$ & $25(10.0)$ & $27(35.1)$ \\
\hline Mycophenolate mofetil & $43(5.3)$ & $4(1.2)$ & $18(7.2)$ & $20(26.0)$ \\
\hline Rituximab & $13(1.6)$ & $2(0.6)$ & $6(2.4)$ & $3(3.9)$ \\
\hline Prednisone & $127(15.6)$ & $41(12.7)$ & $52(20.9)$ & $18(23.4)$ \\
\hline Methotrexate & $53(6.5)$ & $20(6.2)$ & $23(9.2)$ & $6(7.8)$ \\
\hline Azathioprine & $46(5.6)$ & $14(4.3)$ & $12(4.8)$ & $17(22.1)$ \\
\hline Hydroxychloroquine & $60(7.4)$ & $27(8.3)$ & $18(7.2)$ & $2(2.6)$ \\
\hline \multicolumn{5}{|l|}{ Key SSc features } \\
\hline Diffuse cutaneous SSc & $141(18)$ & $32(10)$ & $65(26)$ & $29(38)$ \\
\hline Anticentromere antibody & $499(62)$ & $243(75)$ & $106(43)$ & $8(10)$ \\
\hline Antitopoisomerase I antibody & $92(11)$ & $17(5)$ & $36(14)$ & $30(39)$ \\
\hline Anti-RNA polymerase III antibody & $46(6)$ & $16(5)$ & $24(10)$ & $6(8)$ \\
\hline Modified Rodnan skin score, mean (SD) & $8.0(9)$ & $4.8(7)$ & $6.6(8)$ & $11.7(10)$ \\
\hline Digital ulcers & $301(38)$ & $111(34)$ & $100(40)$ & $38(49)$ \\
\hline Gastrointestinal involvement & $684(84)$ & $261(81)$ & $213(86)$ & $75(97)$ \\
\hline Myopathy & $60(8)$ & $12(4)$ & $25(10)$ & $14(19)$ \\
\hline
\end{tabular}

Definition of abbreviations: HRCT = high-resolution computed tomography; ILD = interstitial lung disease; SSc = systemic sclerosis

Data are shown as $n(\%)$ unless otherwise stated. 
greater than $10 \%$ on HRCT and/or FVC less than $70 \%$, whereas pulmonary arterial hypertension (WHO group 1) was defined as precapillary $\mathrm{PH}$ in the absence of significant ILD, meaning less than $10 \%$ lung fibrosis by HRCT and/or FVC greater than $70 \%$, as previously described $(40,41)$.

\section{Assessment of Standardized Mortality Ratio, Survival Rates, and Causes of Death in SSc-ILD}

For the survival and mortality analyses, all 630 incident patients with SSc from the NorSSc cohort were included, and every death and all the reported causes of death between January 1, 2000, and January 1, 2018, were registered. Vital status at the end of the study was provided by the Norwegian Central Person Register. To calculate the standardized mortality ratio (SMR), Statistics Norway provided a control group from the general Norwegian population with 15 random but sex-, age-, year of birth-, and living area-matched control subjects per patient (12,225 control subjects in total). Additionally, all the 15 control subjects were alive on the date of the matched patient's disease onset (32). The causes of death were based on information

from medical charts, death certificates, and autopsy. When the information from the different sources was not consistent, we used data from the autopsy and medical chart more than data from death certificates. SSc-related deaths were defined as deaths related to progressive, active SSc or deaths caused by organ failures directly related to SSc affection. Deaths unrelated to SSc were of all other causes and divided into several subgroups. If there was no notification on the death certificate or in the patient chart, the cause of death was defined as "unknown" (32).

\section{Statistics}

Analyses were performed with IBM SPSS software, version 25, and STATA software, version 15. Descriptive statistics were applied. Comparisons between groups were evaluated with the Pearson chi-square test, the Fisher exact test, and the KruskalWallis $t$ test, as appropriate. For analyzing correlations, Pearson or Kendall tau-b coefficients were applied, as appropriate. SMRs were calculated as the ratio of the number of deaths among the patients to the number of deaths among their control subjects, representing the Norwegian background population. In the analyses of diagnosis-specific SMRs, the number of deaths was divided by the person-time under observation for each diagnosis studied. Cumulative survival rates at 1,5 , and 10 years after ILD diagnoses were computed by the Kaplan-Meier method and significance was tested with the log-rank test. Items with significant effects on mortality assessed by expert opinion were entered into the Cox proportional hazards model and mortality was expressed by hazard ratio (HR) with its 95\% confidence interval $(\mathrm{CI})$. The multivariable analyses were preceded by estimation of correlation between risk factors. The multivariable models were evaluated by the $\mathrm{C}$-index (values .0 .7 were considered as acceptable) (38).

\section{Results}

\section{Nor-SSc Cohort}

In the Nor-SSc cohort, 815 patients were included, with 630 (77\%) defined as incident SSc cases and $185(23 \%)$ as prevalent SSc cases. From this total, $682(84 \%)$ of the patients were female and $629(77 \%)$ had

A

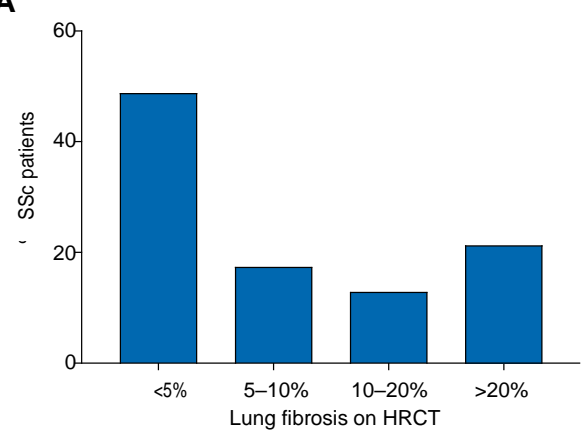

B

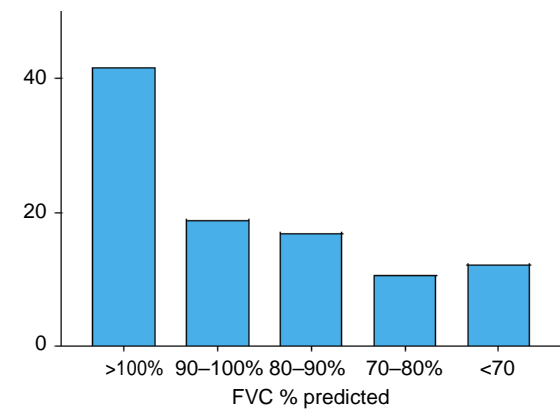

limited cutaneous SSc with a mean age at SSc diagnosis of 53 years (Table 1). Involvement of organ systems other than skin was highly frequent (Table 1). Cumulative treatment with immune modulating drugs is shown in Table 1.

\section{ILD at Baseline}

A baseline lung HRCT was available in $650(80 \%)$ of all Nor-SSc cohort patients. The 650 patients who had undergone HRCT at baseline were more often diffuse cutaneous SSc $(21 \%)$ than the 185 patients with no baseline lung HRCT (8\%, P , $0.001)$, and they were more often positive for antitopoisomerase antibodies (13\% vs. $5 \%, \mathrm{P}=0.010)$ or anti-RNA polymerase III antibody (7\% vs. $0 \%, \mathrm{P}, 0.002)$. Demographics and clinical characteristics did not differ between the patient group with no HRCT conducted at baseline $(\mathrm{n}=$ 185 ) and the patient group with no signs of ILD at the baseline HRCT $(n=326)$.

Analyses of the baseline HRCT images identified varying extent of lung fibrosis in 324 out of 650 patients $(50 \%)$ as shown in Figure 1A. Honeycombing was evident in
C

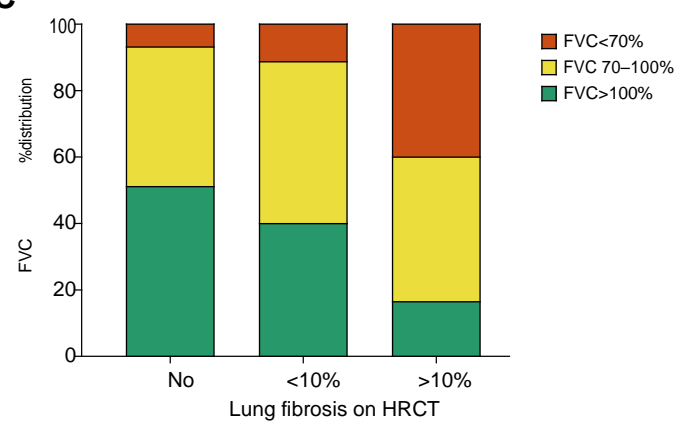

Figure 1. Frequency and distribution of interstitial lung disease in the total Norwegian systemic sclerosis (SSc) cohort $(n=815)$. (A) Patients stratified by extent of lung fibrosis on highresolution computed tomography (HRCT). (B) Patients stratified by percentage of predicted FVC. (C) Distribution of percentage of predicted FVC in patient subsets with no lung fibrosis, less than $10 \%$, or greater than $10 \%$ lung fibrosis. 
63 subjects (10\%), ground-glass opacities in 91 patients $(14 \%)$, and $133(21 \%)$ had bronchiectasis (Table 2).

Baseline PFTs were available in 703 $(86 \%)$ of the Nor-SSc patients. These patients were younger at disease onset than the 112 patients with no PFT available (53 vs. $56 \mathrm{yr}, \mathrm{P}=0.012$ ) and were more likely to be anticentromere antibody-positive (71\% vs. $61 \%, P=0.045)$; otherwise, no significant differences were identified.

The mean FVC predicted at baseline was 94\% (SD, 20.9\%; Table 1) and nearly half of the patients (42\%) had FVC greater than $100 \%$ (Figure 1B). The mean DeCO at baseline was $69 \%$ (SD, 20.2\%).

Proportionate distribution of FVC values at baseline differed between patients who had no lung fibrosis, less than $10 \%$ fibrosis, and greater than $10 \%$ extent of lung fibrosis at the baseline HRCT (Figure 1C and Figure $\mathrm{E} 1$ in the online supplement).

Table 2. Presence and Progression of Interstitial Lung Disease in the Prevalent Norwegian Systemic Sclerosis Cohort $(\mathrm{N}=815)$ Evaluated by HRCT Analyses and PFTs at Baseline and Follow-up

\begin{tabular}{|c|c|c|}
\hline Imaging and Lung Function Characteristics & Baseline & Follow-up \\
\hline \multicolumn{3}{|l|}{ Analyses of lung HRCT } \\
\hline HRCT scan available & $650(80)$ & $460(56)$ \\
\hline Time from baseline to follow-up, yr, mean (SD) & - & $3.7(2.7)$ \\
\hline \multicolumn{3}{|l|}{ Main HRCT findings } \\
\hline Fibrosis & $324(50)$ & $238(52)$ \\
\hline Honeycombing & $63(10)$ & $36(8)$ \\
\hline Ground-glass opacities & $91(14)$ & $59(13)$ \\
\hline Bronchiectasis & $133(21)$ & $92(20)$ \\
\hline Extent of fibrosis, \%, mean (SD) & $10.9(14.2)$ & $13.8(16.2)$ \\
\hline Fibrosis progression & & $105(31)$ \\
\hline Fibrosis progression, \%, mean (SD) & - & $6.2(7.2)$ \\
\hline Patients with $.5 \%$ fibrosis progression & - & $43(18)$ \\
\hline Patients with $.10 \%$ fibrosis progression & - & $18(8)$ \\
\hline \multicolumn{3}{|l|}{ Analyses of PFTs } \\
\hline PFT data available & $703(86)$ & $391(48)$ \\
\hline Time from baseline to follow-up, yr, mean (SD) & - & $6.2(4.2)$ \\
\hline FVC, \% predicted, mean (SD) & $94(20.9)$ & $90(17.3)$ \\
\hline $\begin{array}{l}\text { FVC }, 70 \% \\
\text { New onset FVC ,70\% }\end{array}$ & $90(13)$ & $45 \overline{(13)}$ \\
\hline Total FVC decline, \%, mean (SD) & - & $24.3(17.6)$ \\
\hline Annual FVC decline, \%, mean (SD) & - & $20.8(5.7)$ \\
\hline Dıco, \% predicted, mean (SD) & $69.4(20.2)$ & $56.7(21.1)$ \\
\hline DLCO, $60 \%$ & $217(32)$ & - \\
\hline New-onset Dıco, $60 \%$ & - & $85(35)$ \\
\hline Total Dıco decline, \%, mean (SD) & - & $29.7(14.2)$ \\
\hline Annual DLco decline, $\%$ mean (SD) & - & $21.8(3.9)$ \\
\hline \multicolumn{3}{|l|}{ Course of ILD } \\
\hline Stable FVC & - & $213(54)$ \\
\hline Moderate ILD progression & - & $50(13)$ \\
\hline Severe ILD progression & - & $128(33)$ \\
\hline
\end{tabular}

Definition of abbreviations: HRCT = high-resolution computed tomography; ILD = interstitial lung disease; $\mathrm{PFT}=$ pulmonary function test.

Data are shown as $n(\%)$ unless otherwise stated.
Progression of ILD during the Observation Period

Of all Nor-SSc patients, 460 (56\%) had follow-up lung HRCT images available after a mean 3.7 years (SD, $2.7 \mathrm{yr}$ ) (Table 2 ). These patients had more frequently diffuse cutaneous SSc than patients without follow-up HRCT (13\% vs. 6\%, P , $0.001)$ and were more often positive for antitopoisomerase antibodies (16\% vs. $7 \%$, $\mathrm{P}, 0.001)$ or anti-RNA polymerase III antibodies (12\% vs. $0.2 \%, \mathrm{P}, 0.001)$.

Combined analyses of baseline and follow-up HRCT scans showed that the mean extent of lung fibrosis increased from $11 \%$ at baseline to $14 \%$ at follow-up, whereas frequencies of bronchiectasis, ground-glass opacities, and honeycombing remained stable (Table 2).

Of the 703 patients with PFT at baseline, 391 (48\%) had follow-up PFTs after a mean 6.2 years (SD, $4.2 \mathrm{yr}$ ). These patients were younger at disease onset than those who did not have follow-up PFTs available (51 vs. 55 years, $P, 0.001)$ and they had higher DLCO $\%$ at baseline $(72 \%$ vs. $67 \%, \mathrm{P}, 0.001)$ but comparable FVC and lung fibrosis extent. The mean FVC\% at follow-up was $90 \%(\mathrm{SD}, 25.1 \%)$ and

DLCO $\%$ was $60 \%$ (SD, 21.1\%)

(Table 2).

During the observation period, 128 out of 391 patients (33\%) displayed PFT declines equivalent to severe ILD progression, whereas 50 out of 391 (13\%) showed moderate ILD progression. The patients who displayed PFT declines did not differ from those with stable FVC regarding baseline PFTs or lung fibrosis extent by HRCT. In the subgroup of patients with lung fibrosis less than $10 \%$ and FVC $80 \%$ to $100 \%, 37$ out of 106 $(35 \%)$ had severe ILD progression and 14 out of 106 (14\%) had moderate ILD progression.

We identified 91 Nor-SSc patients with precapillary PH diagnosed by RHC. Of these, 57 had pulmonary arterial hypertension $(7 \%)$, whereas 34 (4\%) had developed PH-ILD. Of all patients with SSc with greater than $10 \%$ lung fibrosis, 40 out of 77 (52\%) had undergone an RHC and 25 out of 40 (63\%) were diagnosed with PH-ILD, giving a cumulative frequency of $32 \% \mathrm{PH}-$ ILD in patients with SSc-ILD with lung fibrosis greater than $10 \%$. Patients with PH-ILD had baseline FVC of 70\% (SD, $19 \%$ ), baseline DLCO 44\% (SD, 17\%), and extent of baseline lung fibrosis $33 \%$ (SD, $22 \%$ ). PH-ILD was diagnosed at a mean of 2.3 years $(\mathrm{SD}, 2.2 \mathrm{yr}$ ) after the baseline HRCT had identified ILD changes.

\section{The Impact of ILD on Survival and} Mortality

In total, there were 169 deaths among the 630 incident Nor-SSc patients (27\%) during a mean 10.1 years (SD, $4.7 \mathrm{yr}$ ) observation period, corresponding to an overall SMR of 2.4 (Figure 2A). Baseline lung HRCT was available in 519 out of 630 incident patients (82\%), and separate analyses of these 519 patients showed that the SMR correlated with presence and extent of lung fibrosis, from SMR 2.2 in patients with no fibrosis to SMR 8.0 in patients with greater than $25 \%$ lung fibrosis (Figure 2B). Correspondingly, we found that the SMR changed across patient groups stratified by baseline FVC\%, with increased mortality evident already in 
A
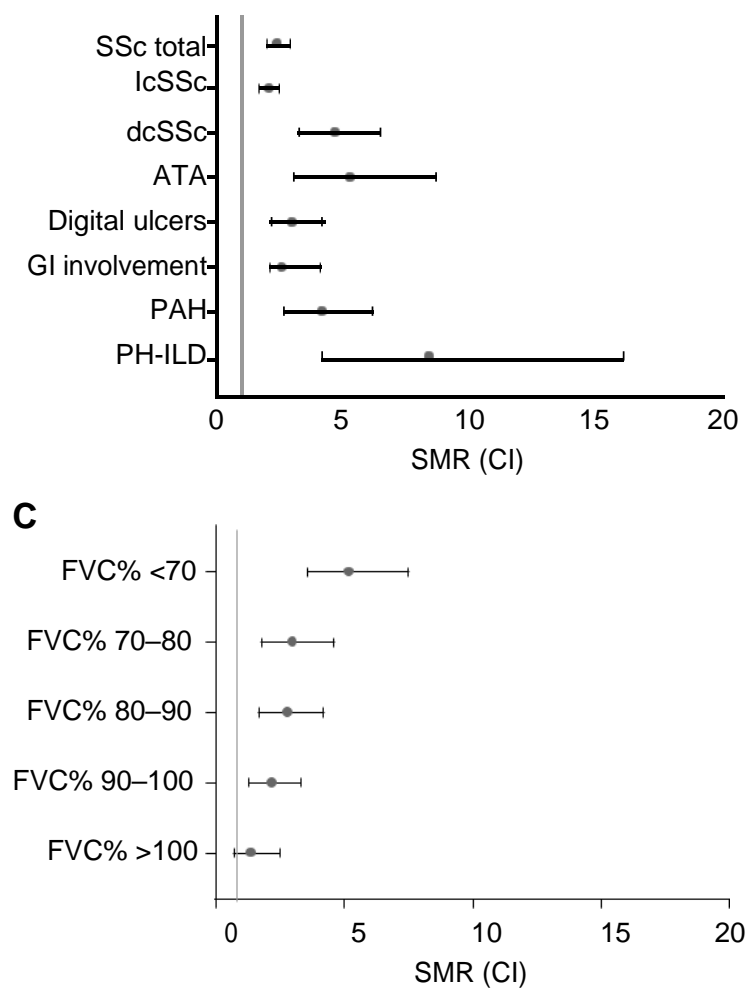

B

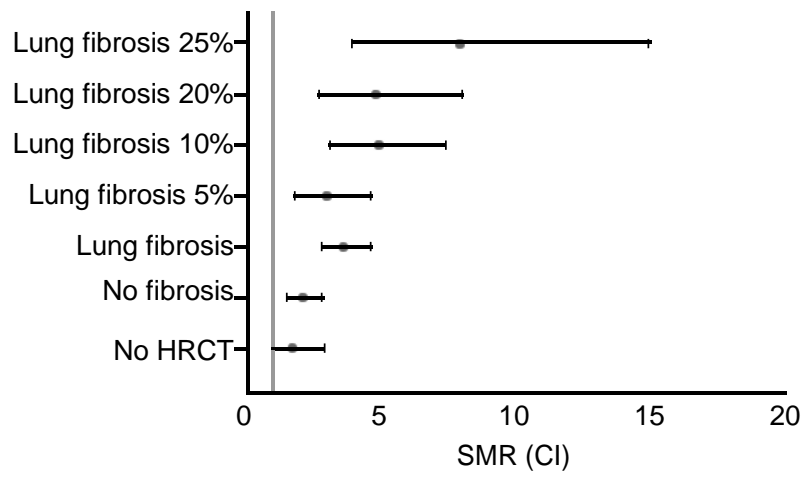

Figure 2. Standardized mortality ratios (SMRs) in the incident Norwegian systemic sclerosis (SSc) cohort ( $\mathrm{n}=630)$. (A) SMRs of SSc, stratified by subgroups and different organ involvements. (B) Extent of lung fibrosis. (C) Percentage of predicted FVC. ATA = antitopoisomerse I antibody; $\mathrm{Cl}=$ confidence interval; dcSSc = diffuse cutaneous SSc; GI = gastrointestinal; HRCT = high-resolution computed tomography; IcSSc = limited cutaneus SSc; PAH = pulmonary arterial hypertension; PH-ILD = pulmonary hypertension caused by interstitial lung disease.

the group having FVC $90 \%$ to $100 \%$ at baseline (Figure 2C).

Survival analyses performed in the patient group who had baseline $\mathrm{FVC} \%$ within the lower normal range (FVC 80-100\%) showed that patients with both limited $(, 10 \%)$ and more extensive lung fibrosis $(.10 \%)$ at baseline had significantly decreased survival compared with the subects patients who had no lung fibrosis on the baseline HRCT $(63 \%$ and $62 \%$ compared with $82 \%, \mathrm{P}=0.010$ ). Cumulative survival curve in the patient group with lower normal-range baseline FVC\% stratified by presence or absence of lung fibrosis is shown in Figure 3.

Progression of lung fibrosis by HRCT was not associated with decreased survival, but ILD progression defined by PFT decline was associated with decreased survival compared with stable FVC with survival rates at 1,5 , and 10 years of $96 \%$, $79 \%$, and $59 \%$ versus $100 \%, 85 \%$, and $78 \%$, respectively $(P=0.018)$ (Figure 4).

Causes of death were compared between incident Nor-SSc patients with and without lung fibrosis by HRCT. In total, 90 out of 233 patients (39\%) with lung fibrosis and 53 out of 284 (19\%) without lung fibrosis died. In addition, we found that the frequency of death causes related to SSc was highest in patients with greater than $10 \%$ fibrosis at baseline (Table E1 in the online supplement).

Univariable parameters associated with mortality in all patients assessed with HRCT at baseline $(n=519)$ are shown in Table E2 in the online supplement. In the multivariable model, age (HR, 1.1; 95\% CI, 1.06-1.11; P ,0.001), sex (HR, 1.9; 95\% CI,

1.15-3.29; $\mathrm{P}=0.013)$, skin involvement evaluated by the Rodnan skin score (HR, 1.04; 95\% CI, 1.01-1.06; $\mathrm{P}=0.001)$, baseline FVC (HR, 0.98; 95\% CI, 0.97-0.99; $\mathrm{P}=0.027)$, baseline lung fibrosis ( $\mathrm{HR}$, 1.03; 95\% CI, 1.01-1.04; $\mathrm{P}=0.002)$, FVC decline (HR, 0.97; 95\% CI, 0.95-0.98; P , 0.001 ), and systolic pulmonary arterial pressure (HR, 1.03; 95\% CI, 1.01-2.04; P $, 0.001)$ were associated with mortality in patients with SSc who had undergone a baseline HRCT (C-index 0.87).
Fibrosis progression and ILD pattern on HRCT, smoking, and immune-modulating treatment were not significantly associated with mortality in multivariable models.

\section{Discussion}

ILD represents a major challenge in SSc but there are to date no precise, populationbased data on its overall impact, limiting opportunities for proper development of ILD screening and management strategies. Here, we assessed ILD by HRCTs and PFTs in a unique nationwide SSc cohort, and report for the first time that the mere presence of ILD at baseline by these modalities has an impact on outcome. Importantly, the findings of reduced survival in patient groups with mild lung fibrosis and normal-range FVC\%, strongly suggest that all patients with SSc should undergo baseline PFT and lung HRCT screening to diagnose ILD early and tailor further management. 


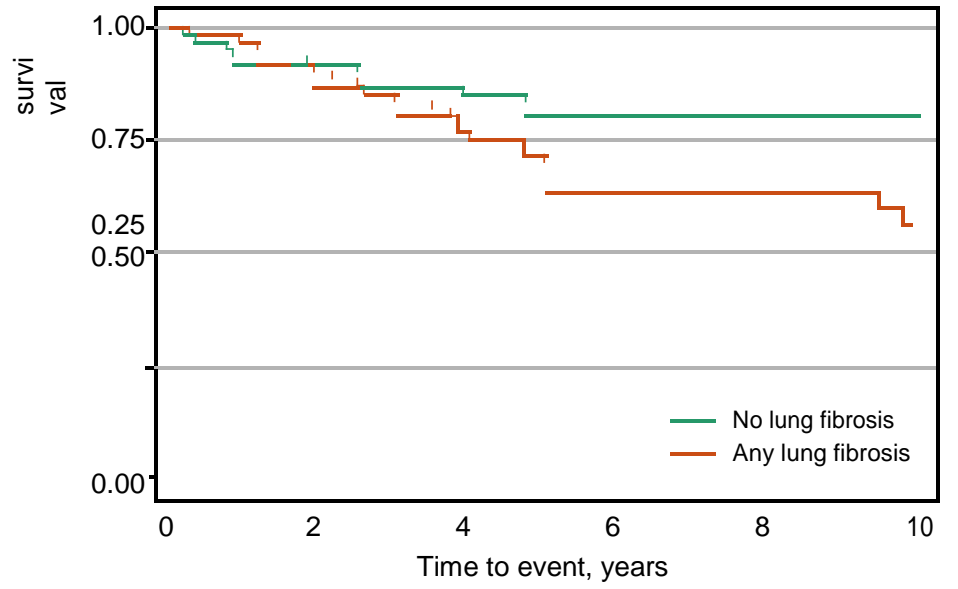

Number at risk
No lung fibrosis 61
Any lung fibrosis 61

$\begin{array}{lllll}55 & 50 & 45 & 35 & 20 \\ 55 & 45 & 36 & 29 & 14\end{array}$

Figure 3. Cumulative survival rates at 1,5 , and 10 years after interstitial lung disease diagnosis shown by Kaplan-Meier curves. Time to death in patients with normal-range FVC\% (80-100\%) stratified by presence or absence of lung fibrosis by high-resolution computed tomography $(P, 0.001)$.

In this study, results from lung imaging by HRCT was available in about $80 \%$ of the Nor-SSc cohort patients, and half of them had parenchymal changes consistent with ILD. Obviously, we do not know the frequency of ILD in the $20 \%$ in whom HRCT was not performed. However, the fact that the demographics, clinical characteristics, and survival of these patients were similar to the group who had no signs of ILD on HRCT argues against the possibility that the cohort subset with missing HRCT should have (severe) unrecognized lung disease. Hence, it is likely that the cumulative incidence of ILD in the Nor-SSc cohort is between 40\% (as it would be if no patient with missing HRCT had ILD) and $50 \%$ (if mild ILD was present in half of the missing HRCT cohort). This estimate is lower than the $70 \%$ to $90 \%$ reported by others but probably reflects differences in cohort selection rather than differing ILD frequencies $(42,43)$. Compared with the study populations in Scleroderma Lung Studies I and II, there were few Nor-SSc cohort patients who displayed ground-

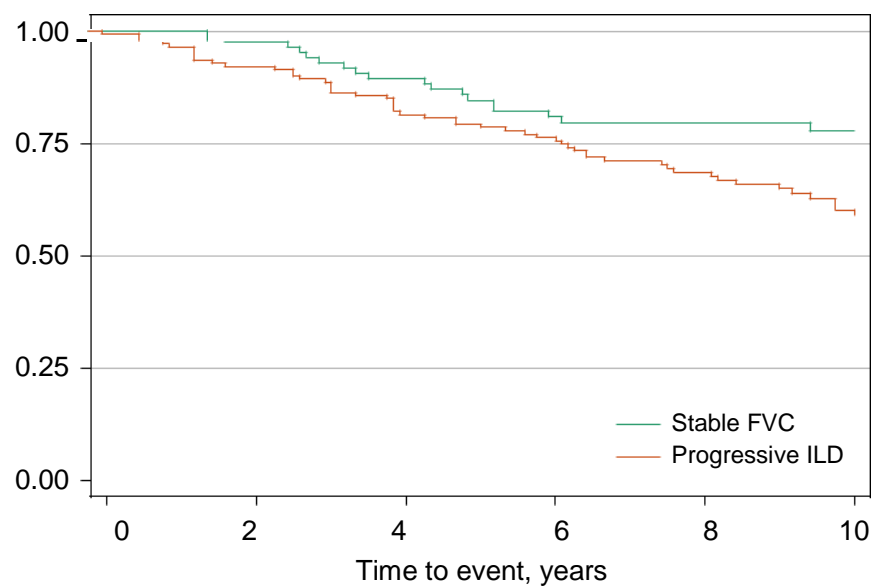

\section{Number at risk \\ Stable FVC 87 \\ Progressive ILD 141}

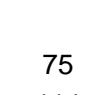

114

85
130

\begin{abstract}
64
102
\end{abstract}

$\begin{array}{ll}52 & 34 \\ 79 & 48\end{array}$

Figure 4. Cumulative survival rates at 1,5 , and 10 years during the observation period shown by Kaplan-Meier curves. Time to death in systemic sclerosis-interstitial lung disease (ILD) patient subsets with stable FVC or ILD progression assessed by pulmonary function tests $(P=0.030)$. glass opacities by lung HRCT $(44,45)$. This may be because we defined superimposed ground-glass opacities as equivalent to fibrosis and therefore underestimated it, or it could be less frequent due to the unselected nature of our cohort compared with randomized clinical trials enriching for more severe ILD.

In line with previous studies $(20,21)$, we identified lung fibrosis at baseline in a large proportion of patients with normalrange PFT values and, in a substantial number, the extent of fibrosis was greater than $10 \%$. This finding underlines that a baseline PFT is not sufficient as a standalone screening tool for SSc-ILD but must be supplemented with additional information from premorbid PFT assessments and/or lung imaging modalities adequate for ILD detection.

Novel to this study, we report that lung fibrosis per se confers increased mortality risk in SSc, and that the SMR is correlated to extent of fibrosis. The finding of highest SMRs in patient with more than $10 \%$ fibrosis corroborates earlier studies showing that extensive ILD (.20\% lung fibrosis on visual scoring), as well as intermediate lung fibrosis (10-30\% lung fibrosis on visual scoring), in combination with FVC less than $70 \%$ in patients with $\mathrm{SSc}$, was associated with increased mortality $(18,46)$.

The results from this study indicate, for the first time, that there is an inverse relationship between mortality risk and baseline FVC\% reductions within the lower range of normal values. We also show that

patients with mild lung fibrosis and normalrange FVC develop frequently progressive ILD. These finding suggest that normal-range FVC values at baseline should not be used as predictors of good disease outcome.

However, as shown in the Nor-SSc cohort survival analyses, it appears that the combination of baseline normal-range FVC and no fibrosis on HRCT confers better prognosis. Hence, it appears rational to suggest that a detailed, combined approach, including extent of fibrosis by HRCT (rather than visual scoring cutoff) and PFTs, should be conducted at baseline in SSc to identify ILD early, assess risk, initiate treatment when indicated, and tailor further management.

Like previously reported in SSc-ILD patients from randomized clinical trials and SSc cohorts from expert centers, we find that FVC decline predicts worse outcome, 
underbuilding the general relevance of the observations from this nationwide, unselected SSc cohort $(19,47)$.

Unfortunately, because we did not have 12month follow-up lung function data available from our cohort, we could not estimate prevalence of progressive fibrosing ILD $(36,47)$. However, we did estimate ILD progression across the entire observation period and found a clear association with worse outcome, even in moderate ILD progression, with long disease duration. Thus, it appears critical to perform regular PFTs in all patients with SSc and to pay close attention to trending declines, particularly in cases with known SSc-ILD at baseline. The role of serial HRCT scans during follow-up is less clear, and prospective studies are required for further evaluation (16). With new treatment options for SSc in pipeline, the interest in developing valid methods for standardized follow-up of patients has increased to be able to diagnose disease progression at an early stage and to be able to start treatment in a timely matter to improve outcome $(48,49)$.

Of the patients with SSc-ILD with lung fibrosis extent greater than $10 \%$, as much as one-third (32\%) were diagnosed with PH-ILD during follow-up. Previous studies have indicated that PH-ILD has poor prognosis $(50,51)$. This notion is substantiated by the highly increased mortality (SMR .8) of Nor-SSc patients with PH-ILD. It may be possible that early detection improves prognosis and patients with SSc-ILD should therefore be screened for $\mathrm{PH}$ on a regular basis.

The main strength of this study is the unique population-based data set. We have previously performed population-based SSc work on a small scale $(31,32)$, but this is the first study ever that is nationwide, has complete patient coverage, and includes comprehensive assessment of ILD by serial HRCT scans and lung function tests. Through labor-intensive, detailed review of each patient's chart, and performance of HRCT image analyses at high resolution, we established multidimensional ILD data from baseline and follow-up. This allowed us to estimate SSc-ILD progression in a large proportion of the patients. Due to the health system in Norway, we did not have any loss to follow-up, and every patient was matched for sex and age with more than 12,000 control subjects drawn from the national population registry to assess the impact of ILD on mortality.

The limitations of the study were that we did not have complete coverage of longitudinal data in all the SSc-ILD cohort patients and no 12-month data were available to assess short-term progression. Also, we might have missed patients with very mild forms of the disease, who had not performed PFTs and HRCT scans, although, as discussed, we assume this issue to have had minor influence on the results. Also, treatment data should be interpreted with caution. Data regarding treatment indication, date of initiation, length of treatment, and cumulative dosage were not available. It is also possible that therapy could represent a confounder on mortality, but, to the best of our knowledge, it has never been shown that medical treatment (with the exception of hematopoietic stem cell transplantation) has any impact on SScrelated mortality.

In conclusion, the results from this population-based SSc cohort study provide new, unbiased data regarding the prevalence and impact of ILD in SSc. Our results indicate a dose-response relationship between lung fibrosis extent and SMR, and between FVC and SMR, evident even in groups with mild lung fibrosis and within normal-range FVC. Taken together, the results from this study suggest that all patients with SSc should be screened with PFT and HRCT at baseline to diagnose ILD early and tailor further management. $n$

Author disclosures are available with the text of this article at www.atsjournals.org.

Acknowledgment: The authors thank all departments of rheumatology in Norway for the collaboration and for providing access to patient data.

\section{References}

1. Allanore Y, Denton C, Matucci-Cerinic M. Systemic sclerosis. In: Bijlsma JWJ, editor. EULAR textbook on rheumatic diseases. London: BMJ Group; 2012.

2. Pattanaik D, Brown M, Postlethwaite BC, Postlethwaite AE. Pathogenesis of systemic sclerosis. Front Immunol 2015;6:272.

3. Denton CP, Khanna D. Systemic sclerosis. Lancet 2017;390:16851699.

4. Elhai M, Meune C, Boubaya M, Avouac J, Hachulla E, Balbir-Gurman A, et al.; EUSTAR Group. Mapping and predicting mortality from systemic sclerosis. Ann Rheum Dis 2017;76:1897-1905.

5. Steen VD, Medsger TA. Changes in causes of death in systemic sclerosis, 1972-2002. Ann Rheum Dis 2007;66:940-944.

6. Frantz C, Avouac J, Distler O, Amrouche F, Godard D, Kennedy AT, et al. Impaired quality of life in systemic sclerosis and patient perception of the disease: a large international survey. Semin Arthritis Rheum 2016; 46:115-123.

7. Nihtyanova SI, Schreiber BE, Ong VH, Rosenberg D, Moinzadeh P, Coghlan JG, et al. Prediction of pulmonary complications and longterm survival in systemic sclerosis. Arthritis Rheumatol 2014;66:1625-1635.

8. Denton CP, Wells AU, Coghlan JG. Major lung complications of systemic sclerosis. Nat Rev Rheumatol 2018;14:511-527.

9. Allanore Y, Simms R, Distler O, Trojanowska M, Pope J, Denton CP, et al. Systemic sclerosis. Nat Rev Dis Primers 2015;1:15002.
10. Kafaja S, Clements PJ, Wilhalme H, Tseng CH, Furst DE, Kim GH, et al. Reliability and minimal clinically important differences of forced vital capacity: results from the Scleroderma Lung Studies (SLS-I and SLSII). Am J Respir Crit Care Med 2017.

11. Kowal-Bielecka O, Fransen J, Avouac J, Becker M, Kulak A, Allanore Y, et al.; EUSTAR Coauthors. Update of EULAR recommendations for the treatment of systemic sclerosis. Ann Rheum Dis 2017;76:1327-1339.

12. Wells AU, Hansell DM, Rubens MB, King AD, Cramer D, Black CM, et al. Fibrosing alveolitis in systemic sclerosis: indices of lung function in relation to extent of disease on computed tomography. Arthritis Rheum 1997;40:1229-1236.

13. Wells AU. High-resolution computed tomography and scleroderma lung disease. Rheumatology (Oxford) 2008;47:v59-v61.

14. Launay D, Remy-Jardin M, Michon-Pasturel U, Mastora I, Hachulla E, Lambert M, et al. High resolution computed tomography in fibrosing alveolitis associated with systemic sclerosis. J Rheumatol 2006;33: 1789-1801.

15. Winklehner A, Berger N, Maurer B, Distler O, Alkadhi H, Frauenfelder T. Screening for interstitial lung disease in systemic sclerosis: the diagnostic accuracy of HRCT image series with high increment and reduced number of slices. Ann Rheum Dis 2012;71: 549-552.

16. Hoffmann-Vold AM, Aaløkken TM, Lund MB, Garen T, Midtvedt $\varnothing$, Brunborg C, et al. Predictive value of serial high-resolution computed tomography analyses and concurrent lung function tests in systemic sclerosis. Arthritis Rheumatol 2015;67:2205-2212. 
17. Wu W, Jordan S, Becker MO, Dobrota R, Maurer B, Fretheim H, et al. Prediction of progression of interstitial lung disease in patients with systemic sclerosis: the SPAR model. Ann Rheum Dis 2018;77:1326-1332.

18. Moore OA, Goh N, Corte T, Rouse H, Hennessy O, Thakkar V, et al. Extent of disease on high-resolution computed tomography lung is a predictor of decline and mortality in systemic sclerosis-related interstitial lung disease. Rheumatology (Oxford) 2013;52:155-160.

19. Goh NS, Hoyles RK, Denton CP, Hansell DM, Renzoni EA, Maher TM, et al. Short-term pulmonary function trends are predictive of mortality in interstitial lung disease associated with systemic sclerosis. Arthritis Rheumatol 2017;69:1670-1678.

20. Molberg $\varnothing$, Hoffmann-Vold AM. Interstitial lung disease in systemic sclerosis: progress in screening and early diagnosis. Curr Opin Rheumatol 2016;28:613-618.

21. Suliman YA, Dobrota R, Huscher D, Nguyen-Kim TD, Maurer B, Jordan S, et al. Brief report: pulmonary function tests: high rate of false-negative results in the early detection and screening of scleroderma-related interstitial lung disease. Arthritis Rheumatol 2015;67:3256-3261.

22. Assassi S, Sharif R, Lasky RE, McNearney TA, Estrada-Y-Martin RM, Draeger H, et al.; GENISOS Study Group. Predictors of interstitial lung disease in early systemic sclerosis: a prospective longitudinal study of the GENISOS cohort. Arthritis Res Ther 2010;12:R166.

23. Gilson M, Zerkak D, Wipff J, Dusser D, Dinh-Xuan AT, Abitbol V, et al. Prognostic factors for lung function in systemic sclerosis: prospective study of 105 cases. Eur Respir J 2010;35:112-117.

24. Plastiras SC, Karadimitrakis SP, Ziakas PD, Vlachoyiannopoulos PG, Moutsopoulos HM, Tzelepis GE. Scleroderma lung: initial forced vital capacity as predictor of pulmonary function decline. Arthritis Rheum 2006;55:598-602.

25. Quanjer PH, Stanojevic S, Cole TJ, Baur X, Hall GL, Culver BH, et al.; ERS Global Lung Function Initiative. Multi-ethnic reference values for spirometry for the 3-95-yr age range: the global lung function 2012 equations. Eur Respir J 2012;40:1324-1343.

26. Flaherty KR, Mumford JA, Murray S, Kazerooni EA, Gross BH, Colby TV, et al. Prognostic implications of physiologic and radiographic changes in idiopathic interstitial pneumonia. Am J Respir Crit Care Med 2003;168:543-548.

27. Jegal Y, Kim DS, Shim TS, Lim CM, Do Lee S, Koh Y, et al. Physiology is a stronger predictor of survival than pathology in fibrotic interstitial pneumonia. Am J Respir Crit Care Med 2005;171:639-644.

28. Latsi PI, du Bois RM, Nicholson AG, Colby TV, Bisirtzoglou D, Nikolakopoulou A, et al. Fibrotic idiopathic interstitial pneumonia: the prognostic value of longitudinal functional trends. Am J Respir Crit Care Med 2003;168:531-537.

29. Raghu G, Collard HR, Egan JJ, Martinez FJ, Behr J, Brown KK, et al.; ATS/ERS/JRS/ALAT Committee on Idiopathic Pulmonary Fibrosis. An official ATS/ERS/JRS/ALAT statement: idiopathic pulmonary fibrosis: evidence-based guidelines for diagnosis and management. Am J Respir Crit Care Med 2011;183:788-824.

30. Andr easson' K, Saxne T, Bergknut C, Hesselstrand R, Englund M. Prevalence and incidence of systemic sclerosis in southern Sweden: population-based data with case ascertainment using the 1980 ARA criteria and the proposed ACR-EULAR classification criteria. Ann Rheum Dis 2014;73:1788-1792.

31. Hoffmann-Vold AM, Midtvedt $\varnothing$, Molberg $\varnothing$, Garen T, Gran JT. Prevalence of systemic sclerosis in south-east Norway. Rheumatology (Oxford) 2012;51:1600-1605.

32. Hoffmann-Vold AM, Molberg $\varnothing$, Midtvedt $\varnothing$, Garen T, Gran JT. Survival and causes of death in an unselected and complete cohort of Norwegian patients with systemic sclerosis. J Rheumatol 2013;40:1127-1133.

33. van den Hoogen F, Khanna D, Fransen J, Johnson SR, Baron M, Tyndall A, et al. 2013 classification criteria for systemic sclerosis: an American college of rheumatology/European league against rheumatism collaborative initiative. Ann Rheum Dis 2013;72: 1747-1755.

34. Hansell DM, Bankier AA, MacMahon H, McLoud TC, Muller" NL, Remy J. Fleischner Society: glossary of terms for thoracic imaging. Radiology 2008;246:697-722.

35. Quanjer PHTG, Tammeling GJ, Cotes JE, Pedersen OF, Peslin R Yernault JC. Lung volumes and forced ventilatory flows. Report Working Party Standardization of Lung Function Tests, European
Community for Steel and Coal. Official Statement of the European Respiratory Society. Eur Respir J Suppl 1993;16:5-40.

36. Khanna D, Mittoo S, Aggarwal R, Proudman SM, Dalbeth N, Matteson EL, et al. Connective Tissue Disease-associated Interstitial Lung Diseases (CTD-ILD) - Report from OMERACT CTD-ILD Working Group. J Rheumatol 2015;42:2168-2171.

37. Zappala CJ, Latsi PI, Nicholson AG, Colby TV, Cramer D, Renzoni EA, et al. Marginal decline in forced vital capacity is associated with a poor outcome in idiopathic pulmonary fibrosis. Eur Respir J 2010;35:830-836.

38. Tennøe AH, Murbræch K, Andreassen JC, Fretheim H, Garen T, Gude $\mathrm{E}$, et al. Left ventricular diastolic dysfunction predicts mortality in patients with systemic sclerosis. J Am Coll Cardiol 2018:72:1804-1813.

39. Galie` N, Humbert M, Vachiery JL, Gibbs S, Lang I, Torbicki A, et al.; ESC Scientific Document Group. 2015 ESC/ERS Guidelines for the diagnosis and treatment of pulmonary hypertension: The Joint Task Force for the Diagnosis and Treatment of Pulmonary Hypertension of the European Society of Cardiology (ESC) and the European Respiratory Society (ERS): Endorsed by: Association for European Paediatric and Congenital Cardiology (AEPC), International Society for Heart and Lung Transplantation (ISHLT). Eur Heart J 2016;37:67-119.

40. Hoffmann-Vold AM, Hesselstrand R, Fretheim $\mathrm{H}$, Ueland $\mathrm{T}$, Andreassen AK, Brunborg C, et al. CCL21 as a potential serum biomarker for pulmonary arterial hypertension in systemic sclerosis. Arthritis Rheumatol 2018;70:1644-1653.

41. Hoffmann-Vold AM, Fretheim H, Midtvedt $\varnothing$, Kilian K, Angelshaug M, Chaudhary $A$, et al. Frequencies of borderline pulmonary hypertension before and after the DETECT algorithm: results from a prospective systemic sclerosis cohort. Rheumatology (Oxford) 2018; 57:480-487.

42. Walker UA, Tyndall A, Czirj ak' L, Denton CP, Farge-Bancel D, KowalBielecka O, et al.; EUSTAR co-authors. Geographical variation of disease manifestations in systemic sclerosis: a report from the EULAR Scleroderma Trials and Research (EUSTAR) group database. Ann Rheum Dis 2009;68:856-862.

43. Walker UA, Tyndall A, Czirjak' L, Denton C, Farge-Bancel D, KowalBielecka $\mathrm{O}$, et al. Clinical risk assessment of organ manifestations in systemic sclerosis: a report from the EULAR Scleroderma Trials And Research group database. Ann Rheum Dis 2007;66:754-763.

44. Tashkin DP, Elashoff R, Clements PJ, Goldin J, Roth MD, Furst DE, et al.; Scleroderma Lung Study Research Group. Cyclophosphamide versus placebo in scleroderma lung disease. N Engl J Med 2006;354: 2655-2666.

45. Tashkin DP, Roth MD, Clements PJ, Furst DE, Khanna D, Kleerup EC, et al.; Sclerodema Lung Study II Investigators. Mycophenolate mofeti versus oral cyclophosphamide in scleroderma-related interstitial lung disease (SLS II): a randomised controlled, double-blind, parallel group trial. Lancet Respir Med 2016;4:708-719.

46. Goh NS, Desai SR, Veeraraghavan S, Hansell DM, Copley SJ, Maher TM, et al. Interstitial lung disease in systemic sclerosis: a simple staging system. Am J Respir Crit Care Med 2008;177:1248-1254.

47. Volkmann ER, Tashkin DP, Sim M, Li N, Goldmuntz E, Keyes-Elstein L, et al.; SLS I and SLS II study groups. Short-term progression of interstitial lung disease in systemic sclerosis predicts long-term survival in two independent clinical trial cohorts. Ann Rheum Dis 2019;78:122-130.

48. Khanna D, Distler JHW, Sandner P, Distler O. Emerging strategies for treatment of systemic sclerosis. J Scleroderma Relat Disord 2018;1: 186-193.

49. Ryerson CJ, Denton CP. The fine line between success and failure in scleroderma lung fibrosis trials. Am J Respir Crit Care Med 2018;197: 553-554.

50. Le Pavec J, Girgis RE, Lechtzin N, Mathai SC, Launay D, Hummers LK, et al. Systemic sclerosis-related pulmonary hypertension associated with interstitial lung disease: impact of pulmonary arterial hypertension therapies. Arthritis Rheum 2011;63:2456-2464.

51. Elinoff JM, Agarwal R, Barnett CF, Benza RL, Cuttica MJ, Gharib AM, et al. Challenges in pulmonary hypertension: controversies in treating the tip of the iceberg. A Joint National Institutes of Health Clinical Center and Pulmonary Hypertension Association Symposium Report. Am J Respir Crit Care Med 2018;198: 166-174. 